\title{
Alzheimer's disease and sleep disturbances: a review
}

\author{
Doença de Alzheimer e distúrbios do sono: uma revisão \\ Conrado Regis BORGES ${ }^{1}$, Dalva POYARES ${ }^{2}$, Ronaldo PIOVEZAN², Ricardo NITRINI¹, Sonia BRUCKI ${ }^{1}$
}

\begin{abstract}
The association between Alzheimer's disease (AD) and sleep disturbances has received increasing scientific attention in the last decades. However, little is known about the impact of sleep and its disturbances on the development of preclinical AD stages, such as mild cognitive impairment. This review describes the evolution of knowledge about the potential bidirectional relationships between AD and sleep disturbances exploring recent large prospective studies and meta-analyses and studies of the possible mechanisms through which sleep and the neurodegenerative process could be associated. The review also makes a comprehensive exploration of the sleep characteristics of older people, ranging from cognitively normal individuals, through patients with mild cognitive impairment, up to the those with dementia with AD.
\end{abstract}

Keywords: Alzheimer disease; sleep initiation and maintenance disorders; cognitive dysfunction.

\section{RESUMO}

A associação entre Doença de Alzheimer (DA) e distúrbios do sono vem recebendo atenção crescente nas últimas décadas. No entanto, pouco se sabe sobre o impacto do sono e suas alterações no desenvolvimento de estágios pré-clínicos da doença, como é o caso do Comprometimento Cognitivo Leve (CCL). Esta revisão descreve a evolução do conhecimento sobre as relações potencialmente bidirecionais entre DA e distúrbios do sono, explorando grandes estudos prospectivos e meta-análises, assim como estudos dos possíveis mecanismos da associação entre o sono e as doenças neurodegenerativas. Também revisamos amplamente as características do sono de pessoas idosas, incluindo indivíduos cognitivamente normais, com CCL e com demência por DA.

Palavras-chave: Doença de Alzheimer; distúrbios do início e da manutenção do sono; disfunção cognitiva.

Sleep is very important to the central nervous system (CNS) physiology. Changes in sleep quality and structure take place over a lifetime and are accentuated during healthy aging. Several studies have shown an association between sleep and CNS disorders, such as cerebrovascular disease, Parkinson's disease, multiple sclerosis, epilepsy, headache, pain and, especially, neurodegenerative disorders ${ }^{1}$.

Alzheimer's disease $(\mathrm{AD})$ is the most prevalent neurodegenerative disorder and is associated with sleep problems. Many previous studies have shown that sleep disorders are risk factors for $\mathrm{AD}$. On the other hand, patients with mild cognitive impairment (MCI) and $\mathrm{AD}$ have shown an increased frequency of sleep disorders. This evidence implies an hypothesis of the existence of a bidirectional association between sleep and $\mathrm{AD}^{2}$.

Understanding this interaction is of utmost importance because it would increase knowledge about the role of sleep in the development of $\mathrm{AD}$, and about the impact $\mathrm{AD}$ has on sleep quality. This could offer cues about how sleep interferes with cognitive and behavioural symptoms of dementia. Additionally, understanding the association between $\mathrm{AD}$ and sleep could lead to insights into the pathophysiological mechanisms of $\mathrm{AD}$.

The scientific community has widely discussed sleep disturbances in normal aging. From the late '70s to early '80s, interest in the characteristics of the sleep of patients with

\footnotetext{
1 Universidade de São Paulo, Faculdade de Medicina, Hospital das Clínicas, Departamento de Neurologia, Grupo de Neurologia Cognitiva e do Comportamento, São Paulo SP, Brasil;

2 Universidade Federal de São Paulo, Escola Paulista de Medicina, Instituto do Sono, São Paulo SP, Brasil.

Conrado Regis BORGES (iD) https://orcid.org/0000-0003-3220-7746; Dalva POYARES ID https://orcid.org/0000-0003-0664-0968; Ronaldo PIOVEZAN (iD https://orcid.org/0000-0003-2537-8766; Ricardo NITRINI iD https://orcid.org/0000-0002-5721-1525; Sonia Maria Dozzi BRUCKI iD https://orcid.org/0000-0002-8303-6732

Correspondence: Conrado Regis Borges; R. Enéas de Carvalho Aguiar, 255; 05403-000 São Paulo SP, Brasil; E-mail: conradoborges@outlook.com Conflict of interest: There is no conflict of interest to declare.

Received 03 May 2019; Received in final form 19 June 2019; Received in final form 23 June 2019.
} 
dementia started to increase. Studies using polysomnography to compare $\mathrm{AD}$ patients in the healthy elderly ${ }^{3}$ provided knowledge about sleep-disordered breathing ${ }^{3}$ and changes in sleep structure (especially in rapid eye movement [REM] sleep) in $\mathrm{AD}^{3}$. The REM sleep behavioral disorder was described ${ }^{4}$ late in the 1980s, but its association with Lewy bodies dementia and Parkinson's disease was uncovered only a decade later 5 .

The knowledge in this area has developed impressively, but the direction of the relationship between sleep disturbances and $\mathrm{AD}$ remains unknown. Initially, the dominant hypothesis was that the incidence of both disorders increased with age and they merely coexisted. However, since the beginning of the $21^{\text {st }}$ century, many prospective longitudinal studies have been published, revealing an association of sleep disorders and an increased risk of dementias.

Consequently, studies in animal models and humans were developed to explain how sleep disorders could lead to $\mathrm{AD}$ and other dementias. Initial explanations linked the impaired sleep observed in aged people with higher brain metabolism, which would result in more neuronal damage in the long term and, consequently, in the development of $\mathrm{AD}^{2,6}$. However, this understanding changed dramatically when, in 2012, a group discovered a close association between sleep and the glymphatic system, which is responsible for the clearance of toxic substances from the $\mathrm{CNS}^{7-9}$.

Another recently expanding research topic is the role of sleep disturbances in early phases of $\mathrm{AD}$, such as MCI, especially the amnestic form, subjective cognitive decline and cognitively normal individuals with $\mathrm{AD}$ biomarkers. As these concepts are still recent, few studies addressing their relationship with sleep have been carried out ${ }^{10}$.

\section{SLEEP ASSESSMENT AND COGNITIVE IMPAIRMENT}

The study of sleep disorders demands the conduction of an arsenal of diagnostic methods. Two types of tools are commonly used: subjective and objective. Among the subjective methods are methods registering behaviour and scales of sleep. The main objective methods are polysomnography and actigraphy.

Despite the advantages of subjective methods, such as convenience and reduced costs, these may lead to higher rates of false positive and false negative results, interfering in the clinical management. Some studies have shown a discrepancy between the information obtained by subjective and objective methods. For example, individuals with MCI presented with greater misperception of their sleep onset time than the corresponding healthy elderly ${ }^{11}$.

Actigraphy is a reliable method to assess sleep characteristics, despite being less sensitive than polysomnography. Some of its advantages are the low cost for an objective method, the convenience of execution, the low rate of interference in sleep quality and the possibility of mapping the total motor activity of the participant for longer periods, thus being suitable for circadian cycle studies. Also it is appropriate for diagnosing periodic limb movement syndrome ${ }^{12}$.

On the other hand, polysomnography is a more accurate test for diagnosing multiple kinds of sleep disorders (such as sleep-disordered breathing, periodic limb movement syndrome, and REM sleep behavioural disorder) and alterations in sleep structure. However, it is more challenging to perform because of the higher costs and the need to keep the patients in a sleep laboratory overnight. Moreover, polysomnography does not allow the assessment of habitual sleep patterns, mainly in older adults, who have a higher frequency of circadian rhythm changes and daytime naps. In Table 1, we compare the potential differences and advantages of the one subjective and two objective methods.

\section{SLEEP CHANGES IN NORMAL AGING}

Studies assessing characteristics of sleep in individuals of many age groups have shown that several sleep changes occur in older adults compared with younger ones. Changes in sleep structure occur and progress as the age advances.

\section{Sleep structure and aging}

Sleep quality worsens with aging. Older adults have greater difficulty initiating and, maintaining sleep, presenting with longer sleep time latency ${ }^{13,14}$ and increased sleep fragmentation ${ }^{13-15}$. Thus, they tend to spend more time awake at night (reduced total sleep time) and have reduced sleep efficiency ${ }^{14}$. Indeed, some studies reported the wake time after sleep onset as one of the sleep variables showing a greater age-related effect size in polysomnographic and actigraphic studies ${ }^{16}$.

Table 1. Potential advantages and differences between self-report, actigraphy and polysomnography recordings.

\begin{tabular}{|c|c|c|c|}
\hline \multirow{2}{*}{ Diagnostic methods } & \multirow{2}{*}{ Subjective } & \multicolumn{2}{|c|}{ Objective } \\
\hline & & Actigraphy & Polysomnography \\
\hline Applicability & +++ & ++ & + \\
\hline Costs & + & ++ & +++ \\
\hline Reliability & + & ++ & +++ \\
\hline Disturbed sleep? & & - & +++ \\
\hline Circadian circle & & +++ & + \\
\hline Sleep structure & & - & +++ \\
\hline $\begin{array}{l}\text { Sleep-disordered } \\
\text { breathing }\end{array}$ & & + & +++ \\
\hline $\begin{array}{l}\text { REM sleep behavioral } \\
\text { disorder }\end{array}$ & & + & +++ \\
\hline $\begin{array}{l}\text { Periodic limb } \\
\text { movement syndrome }\end{array}$ & & + & +++ \\
\hline
\end{tabular}


Additionally, sleep-disordered breathing and periodic limb movement syndrome may contribute to worsening these sleep parameters ${ }^{15}$, as well as medical comorbidities, pain, and nocturia, which all have increased prevalence in the elderly.

As aging progresses, the circadian rhythms become weaker and less synchronized ${ }^{17}$. Progressively, the weaker circadian response to external cues blurs the boundaries between asleep and awake ${ }^{18}$. Moreover, advances in sleep stages tend to occur with aging ${ }^{13,15}$. Older adults frequently suffer from sleepiness in the early evening and may wake up too early, becoming unable to return to sleep ${ }^{17}$. With the impairment in the sleep-wake cycle, the frequency of daytime naps and excessive daytime sleepiness increase. This leads to reduced participation in daytime routines and activities of daily living ${ }^{15}$.

Polysomnography studies describe a reduction in slowwave sleep and a compensatory increase in the lighter sleep stages (N1 and N2) in the elderly compared with young and middle-aged people ${ }^{13,14,15,17}$. Figure 1 shows the sleep phases organization with age published by Moraes et al. ${ }^{16}$.

Fewer and shorter non-REM/REM cycles are observed ${ }^{13}$. However, REM sleep reductions are subtler than those observed in non-REM sleep, and fewer awakenings occur in REM sleep. Similarly, a study in the São Paulo general population showed a small reduction in slow-wave sleep and REM sleep and larger effect size for the wake time after sleep onset and respiratory arousals ${ }^{16}$.

Despite these characteristics of sleep in the elderly, it is necessary to consider that individual variability exists. For example, people with the APOE E4 genotype show worse sleep patterns ${ }^{13}$, and the coexistence of medical comorbidities.

\section{Electroencephalographic (EEG) sleep changes}

On a structural level, changes occur in several measures of non-REM sleep in the elderly, such as reductions of the amplitude of slow waves and, consequently, a decrease in slow-wave activity, that is the quantification of the spectral power in the $0.5-4.0 \mathrm{~Hz}$ range in non-REM sleep ${ }^{13,19}$. In young people, the slow-wave activity in the first sleep cycles is usually higher, and decreases exponentially across consecutive sleep cycles. Slow-wave activity is also linked to the homeostatic drive of sleep following continued wakefulness. For example, following sleep deprivation, an increase in slowwave activity occurs, especially in the initial sleep cycles. In aged people, however, this variation is reduced, which suggests an impaired non-REM regulation in this population. These alterations are more prominent in anterior brain regions in comparison with other regions ${ }^{19}$.

Moreover, the amplitude and the density of slow waves are reduced progressively as aging proceeds. Furthermore, there is also an average reduction of the non-REM frequency

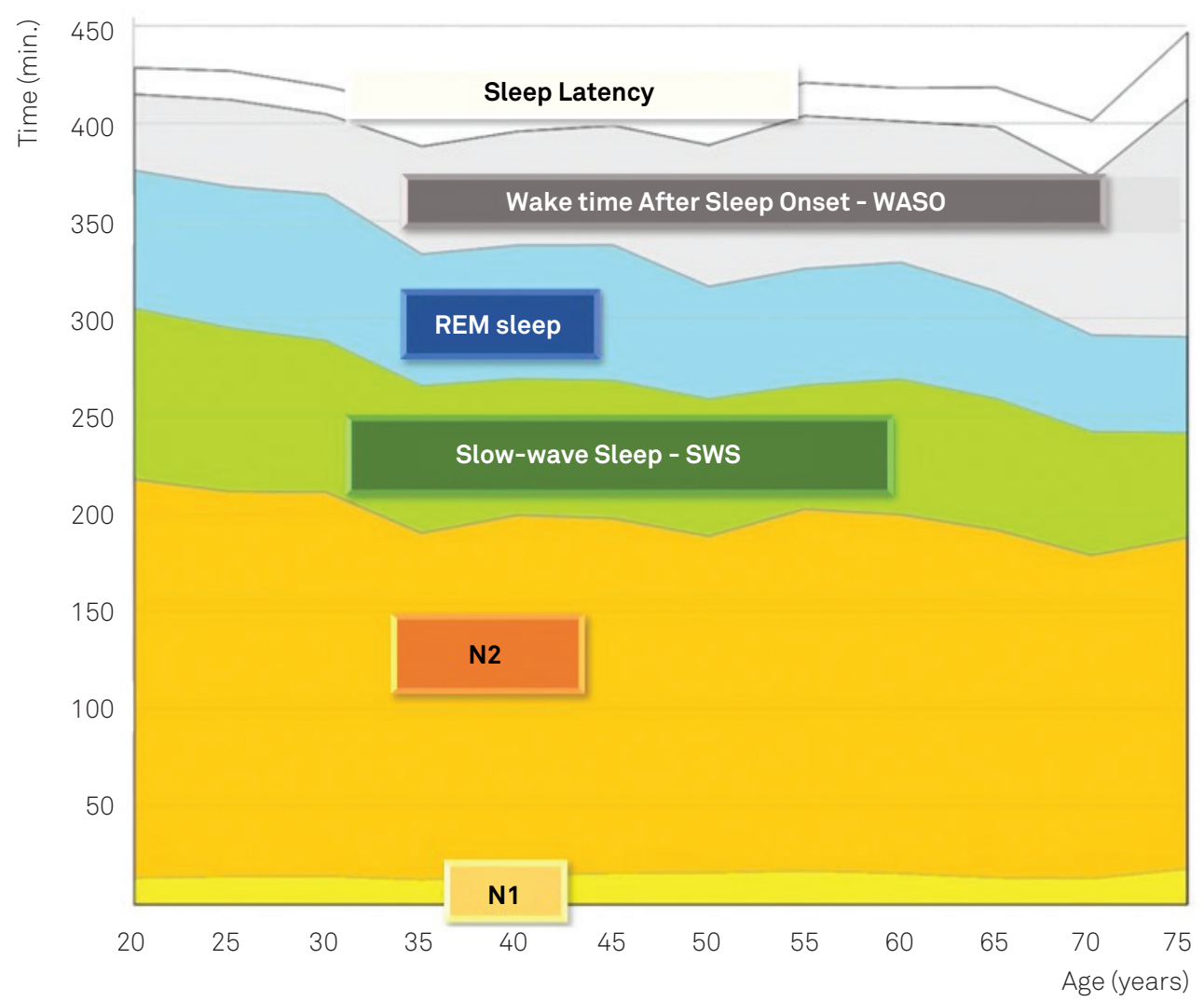

Figure 1. Organization of the sleep in a Brazilian population sample of normal individuals. (The authors authorized the use of the image $)^{16}$. 
of about $0.1 \mathrm{~Hz}$. The brain region that shows more prominent alteration in the non-REM frequencies is the prefrontal region, and there is a reduction in the gray matter, especially in the medial prefrontal cortex and middle frontal gyrus ${ }^{13}$.

The N2 light sleep stage comprises about $45 \%$ to $55 \%$ of the sleep time. One of the most important markers of this stage is the sleep spindles-brief bursts of oscillatory fast activity (with frequencies between $12 \mathrm{~Hz}$ and $16 \mathrm{~Hz}$ ), with waxing and waning amplitudes. They are generated by the thalamus reticularis nucleus neurons and have an association especially with sensorimotor learning, but also with visuospatial and memory functions ${ }^{20}$. Populational EEG studies have revealed that the spectral power in the frequency range of sleep spindles $(12-16 \mathrm{~Hz})$ is also reduced in the elderly ${ }^{13}$. Spindle density and amplitude reductions occur mainly in the frontal lobe regions. In contrast, reductions in spindle durations are maximal over posterior EEG derivations $^{13}$. Altogether, these changes in the sleep spindles generation may be associated with impaired procedural learning observed in the healthy elderly ${ }^{20}$.

\section{Are sleep impairments part of normal aging?}

Whether these sleep changes are part of the normal process of aging or are caused by comorbid conditions is still controversial. For example, psychiatric disorders such as depression and anxiety, pain syndromes, pulmonary, cardiac, neurologic and endocrinological diseases, among others, have consistently been correlated with higher incidences of sleep disturbances in older people ${ }^{17}$. However, healthy elderly adults have also exhibited higher frequencies of sleep disturbances and changes in sleep architecture even after controlling for other variables.

Another controversy is whether aged people need less sleep time or not. Arguments in favour say that older people sleep much less than younger when offered an extended period in bed. Moreover, after sleep-deprivation, aged people suffer from less rebound of non-REM sleep. Furthermore, older individuals present with less somnolence after nonREM sleep deprivation than young ones ${ }^{13}$. However, a recent meta-analysis including 27 cohort studies and more than 70,000 elderly individuals, followed up from 3.4 to 35 years, demonstrated that both long and short sleep duration, as well as daytime napping for 30 minutes or more, were associated with increased all-cause mortality ${ }^{21}$. More recently, a prospective cohort study of community-dwelling elderly without dementia found higher incidence rates of dementia (all-cause dementia, $\mathrm{AD}$ and vascular dementia) among those with short and long sleep durations ${ }^{22}$.

Alternatively, researchers arguing against this thesis claim that old people continue to have a homeostatic sleep drive. They feel less impact from sleep deprivation, presenting with less somnolence due to sleep loss and, instead, showing more cognitive alterations. One possible explanation is a chronic adaptation to the age-related sleep changes. For example, some studies have revealed that older adults without sleep deprivation show a cognitive performance that is similar to that observed in sleep-deprived younger people ${ }^{23}$. Moreover, the disruption of non-REM sleep could explain the worse memory performance shown by the elderly ${ }^{13}$.

Addressing sleep in preclinical $\mathrm{AD}$ is still a gray zone and complex matter. Musiek et al. ${ }^{24}$ performed a cross-section study of 205 cognitively-normal patients, who underwent 7-14 days of wrist actigraphy and assessment of AD biomarkers (such as positron emission tomography (PET)- Pittsburgh Compound B and cerebrospinal fluid biomarkers). The study showed that cognitively healthy people with $\mathrm{AD}$ biomarkers (i.e., preclinical $\mathrm{AD}$ ) had more rest-activity rhythm fragmentation, independent of age or sex. ${ }^{24}$ Studies like this shed some light on the distinction between sleep in healthy aging and the initial phases of $\mathrm{AD}$.

\section{SLEEP CHANGES IN MILD COGNITIVE IMPAIRMENT}

Mild cognitive impairment is characterized by a memory impairment beyond that expected for age and education, although the patients do not manifest critical functional alterations. Cognitive scientists classified MCI as amnestic and non-amnestic MCI, and subdivided each category into unique domains and multiple domains according to neuropsychological evaluations. This classification is important because patients with multiple domain amnestic MCI have a higher rate of conversion to dementia ${ }^{25}$.

Large population studies have linked MCI to an increased rate of sleeping problems compared with healthy people of the same age. The prevalence of sleep disturbances in patients with MCI ranges from $8.8 \%$ to $59 \%{ }^{26}$. Moreover, primary sleep disorders, such as sleep-disordered breathing, periodic limb movement syndrome, restless leg syndrome, and REM sleep behavioural disorder are more common in $\mathrm{MCI}^{27}$. As well, prospective cohort studies have shown associations of sleep disturbances with a higher rate of progression of $\mathrm{MCI}$ to $\mathrm{AD}^{28}$.

A meta-analysis of studies comparing objective sleep measures of patients with MCI with those of the healthy elderly showed that the former had shorter total sleep time, sleep efficiency, cycling alternating pattern (a measure of sleep instability) and longer sleep latency. Patients with nonamnestic MCI, on the other hand, had a greater total sleep time compared with the healthy elderly ${ }^{12}$. Other parameters, such as the wake time after sleep onset, slow-wave sleep, REM latency and REM (\%), and periodic limb movement syndrome did not differ between the groups ${ }^{12}$. However, patients with MCI showed increased night-time behaviors ${ }^{29}$.

A well-designed study compared the cognitive performance of patients with amnestic MCI with that of the healthy elderly using neuropsychological tests. The participants underwent neuropsychological evaluation before and after sleep for two consecutive days and polysomnography 
during the two nights of sleep. In the control group, memory performance increased in the tests after sleep compared with the period before the sleep (in the tests of word pair recall, fact recognition, and object priming). However, memory performance did not improve in participants with amnestic MCI (even decreased in the word pair recall test) after sleep consolidation ${ }^{30}$.

In patients with non-amnestic MCI, sleep problems are also common. Sleep fragmentation has been associated with memory dysfunction and increased wake time after sleep onset with executive dysfunctions (impairments in attention, response inhibition, concept forming, and problemsolving $)^{31}$. Studies assessing sleep architecture revealed that patients with MCI had decreased slow-wave sleep compared with the normal elderly ${ }^{15,30}$ and increased fragmentation of non-REM sleep ${ }^{10}$. Moreover, borderline changes have been found in REM, wake time after sleep onset, REM latency, and sleep efficiency ${ }^{30}$.

The assessment of EEG power across non-REM and REM sleep also revealed differences between patients with amnestic MCI and the healthy elderly. The former showed a reduction in power of delta and theta EEG frequency bands ${ }^{30}$. The same reduction occurred in the fast spindle counts on frontal locations ${ }^{30}$. When correlating cognitive performance and sleep parameters, researchers observed an association of the reductions in delta and theta power with a decreased cognitive performance after sleep ${ }^{30}$. Furthermore, the duration of REM was reduced by $5 \%$ in MCI patients. Increasing REM sleep time improved the performance of immediate recall ${ }^{10}$.

\section{SLEEP CHANGES IN ALZHEIMER'S DISEASE DEMENTIA}

Electroencephalographic studies on $\mathrm{AD}$ patients in the 1980s and 1990s provided valuable information about sleep patterns in $\mathrm{AD}$ dementia compared with the healthy elderly and neuropsychiatric disorders, such as depression. Sleep disturbances in patients with $\mathrm{AD}$ dementia were qualitatively similar to those seen in the healthy elderly ${ }^{32}$. However, the severity of the changes was usually higher, and the REM sleep had specific alterations. Wakefulness commonly increased and, consequently, sleep was more disrupted at night in $\mathrm{AD}$ dementia, with an increase in number and duration of awakenings, resulting in a shorter total sleep time and lower sleep efficiency ${ }^{33}$. As a result, a higher percentage of stage 1 and 2 sleep and lower percentage of slow-wave sleep was observed in these patients ${ }^{34}$.

Additionally, $\mathrm{AD}$ dementia is related to reductions of specific features of the N2 light sleep stage, such as sleep spindles and $\mathrm{K}$ complexes, which become poorly formed, with lower amplitudes and frequencies ${ }^{34}$. These alterations have been reported to worsen with the severity of dementia ${ }^{33,35}$ and, as the disease progresses, distinguishing $\mathrm{N} 1$ and $\mathrm{N} 2$ becomes a challenge. Because of this, some authors classify the sleep without distinguishable stages observed in late $\mathrm{AD}$ as indeterminate nonREM ${ }^{32,34,36}$.

Alzheimer's dementia-related REM sleep alterations may be attributed to dysfunction of the cholinergic system during the evolution of the disease. The basal forebrain degeneration in $\mathrm{AD}$ leads to lower cortical activation during REM sleep ${ }^{32,34}$. Likewise, quantitative EEG studies have shown higher power in theta and delta frequencies, and lower power in alpha and beta frequencies during the REM sleep of patients with $\mathrm{AD}^{34}$. However, the total number of REM sleep episodes and REM sleep latency remain intact ${ }^{34}$.

The first studies evaluating sleep structure in $\mathrm{AD}$ patients were made without segmentation by severity. However, further studies have also been done characterizing sleep in patients with mild stage $\mathrm{AD}$ dementia. Overall, the main change in sleep/wake patterns observed was the marked intrusion of wakefulness into sleep time ${ }^{33}$. In Table 2 we show the sleep characteristics in the healthy elderly, $\mathrm{MCI}$ and $\mathrm{AD}$ patients.

\section{SLEEP DISORDERS AS RISK FACTORS FOR ALZHEIMER'S DISEASE}

Despite some early studies in the $20^{\text {th }}$ century hypothesizing that the sleep disturbances could act as a risk factor to the incidence of dementia, initially, the dominant theory was that both conditions occurred in parallel as aging advanced. This hypothesis changed recently when some prospective cohort studies of incident dementia showed that people with a history of sleep complaints in middle age had an increased risk of developing $\mathrm{AD}$ than those without sleep complaints. Moreover, more recently, meta-analyses have confirmed the potential role of poor sleep and sleep disorders as predictors of $\mathrm{AD}$ development.

\section{Epidemiological studies}

Prospective cohort studies assessing the hypothesis that sleep deterioration and disorders may increase the risk of cognitive impairment and dementia began being published around the year 2000. They provided information on many different aspects of sleep disturbances, and most of them showed an association with the development of dementia. For example, insomnia ${ }^{37}$, excessive daytime sleepiness ${ }^{38,39}$, long sleep duration ${ }^{40,41}$ and short sleep duration ${ }^{40,42,43}$ have been linked to the development of cognitive impairment and/or dementia. Moreover, studies assessing sleep time revealed both short and long sleep times as risk factors, suggesting a U-shaped association between sleep duration and cognitive impairment/dementia ${ }^{40,41}$.

The first studies followed only a few participants and did not use objective methods of assessment of sleep or detailed cognitive evaluation. However, they did show the first results arguing in favour of the association between 
Table 2. Comparative summary of sleep characteristics of healthy elderly, old people with mild cognitive impairment and with AD dementia.

\begin{tabular}{|c|c|c|c|}
\hline Variable & Healthy elderly & Mild cognitive impairment & Alzheimer's dementia \\
\hline Total sleep time & $\downarrow$ & $\downarrow \downarrow$ or $\uparrow$ & $\downarrow \downarrow \downarrow$ or $\uparrow \uparrow$ \\
\hline Sleep efficiency & $\downarrow$ & $\downarrow \downarrow$ & $\downarrow \downarrow \downarrow$ \\
\hline Sleep latency & $\uparrow$ & $\uparrow \uparrow$ & $\uparrow \uparrow$ \\
\hline REM sleep latency & $\uparrow$ & $\uparrow$ & $\uparrow$ \\
\hline Waking after the sleep onset & $\uparrow$ & $\uparrow$ & 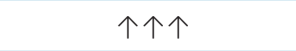 \\
\hline Phase advance & + & ++ & +++ or delay \\
\hline Excessive daytime sleepiness & + & ++ & +++ \\
\hline N1 \& N2 sleep stages & $\uparrow$ & $\uparrow$ & 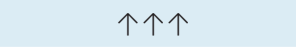 \\
\hline Slow-wave sleep & $\downarrow$ & $\downarrow$ & $\downarrow \downarrow \downarrow$ \\
\hline REM sleep & $\downarrow$ & $\downarrow$ & $\downarrow$ \\
\hline REM cycles (number) & $\downarrow$ & $\downarrow$ & $\downarrow$ \\
\hline REM cycles (duration) & $\downarrow$ & $\downarrow$ & $\downarrow$ \\
\hline Fast waves in REM & $\downarrow$ & $\downarrow$ & $\downarrow \downarrow$ \\
\hline
\end{tabular}

REM: rapid eye movement.

sleep disturbances and cognitive impairment ${ }^{38,44}$. In the early 2000s, cohort studies with a higher number of participants provided more detailed information on the dementia diagnosis and used standardized scales to assess sleep. However, follow-up times of less than five years ${ }^{45}$ prevented reliable conclusions. In 2010, one study with a follow-up between 10 and 14 years showed that sleeping more than nine hours per night was associated with an increased risk of cognitive impairment. An important limitation of this study was the lack of a proper evaluation of dementia, as cognition was assessed only by telephone ${ }^{46}$.

Some of these methodological limitations were addressed the following year by Tranah and colleagues ${ }^{47}$ in a longitudinal study that use wrist actigraphy to assess the sleep and the daily activity of 1,282 healthy community-dwelling women. After 4.9 years of follow-up, the participants underwent a neuropsychological battery and screening for dementia. The authors found that healthy women with impaired circadian rhythms had increased odds of developing dementia and $\mathrm{MCI}$. Further studies corroborated and refined these findings using standardized sleep screening tools and more detailed wrist actigraphy information ${ }^{48}$.

Adding to the findings that change in the circadian rhythms may lead to cognitive disorders, Yaffe et a ${ }^{49}$ demonstrated that healthy women with sleep-disordered breathing were more likely to develop mild cognitive impairment or dementia than women with normal sleep. The study used overnight polysomnography to assess the baseline status of sleep of participants and found sleep-disordered breathing to be a potential risk factor of MCI and dementia. Further polysomnographic studies with larger numbers of participants and with longer follow-ups have confirmed the association between sleep-disordered breathing and incident demen$\mathrm{tia}^{50}$. One of these studies found a reduction of REM sleep, but not non-REM sleep, associated with the incidence of clinical dementia, which could implicate the preponderance of REM sleep-related mechanisms as predictors of dementia ${ }^{50}$.

Despite the progressive methodological improvement of the studies, until 2013, most of the longitudinal studies assessing the incidence of cognitive disorders in patients with sleep disturbances had follow-up times of less than 15 years. This could be considered a limitation because the progression of the $\mathrm{AD}$ pathology precedes the emergence of the cognitive manifestations by $10-15$ years. The first study to address this problem with a median follow-up time of 22.5 years was conducted by Virta and colleagues ${ }^{40}$ in a Finnish cohort of patients aged at least 65 years old. They found that patients with short $(<7$ hours/day) and long ( $>8$ hours/day) sleep times, and with poor sleep quality at baseline, had lower cognitive scores. In 2017, two more studies with similar follow-up times also showed evidence of sleep disturbances as risk factors for the development of dementia $a^{50}$.

Some studies provided additional information. For example, Elwood and colleagues ${ }^{39}$ found an association between sleep disturbances with cognitive impairment non-AD of vascular origin, but not with $\mathrm{AD}$. Potvin and colleagues ${ }^{42}$, in turn, showed that different kinds of sleep disturbances could cause impairments in different cognitive domains (amnestic or nonamnestic) and affected each sex differently. However, the literature available is insufficient to relate specific sleep problems to specific cognitive disorders. Therefore, there is an expectation of more studies designed specifically to this end.

\section{Report on systematic reviews and meta-analyses}

Because of the availability of several epidemiological studies on the association between sleep disturbances and cognitive disorders, recent systematic reviews and metaanalyses have been conducted on this topic. Almondes and collaborators $^{51}$ analysed five community-based prospective 
cohorts aiming to evaluate the risk of dementia in people with a history of insomnia. They found an association of insomnia with a significant risk of all-cause dementia $(\mathrm{RR}=1.53$; 95\% CI 1.07-2.18).

Moreover, Bubu et al..$^{52}$ included 27 studies (69,216 participants) in their meta-analysis. Using broader inclusion criteria and accepting sleep disorders other than insomnia, they found that individuals with sleep problems, in general, had a 1.55 (95\%CI: 1.25-1.93), 1.65 (95\%CI: 1.45-1.86) and 3.78 (95\%CI: 2.27-6.30) times higher risk of $\mathrm{AD}$, cognitive impairment and preclinical $\mathrm{AD}$, respectively. In addition to these findings, they could also estimate an average magnitude of effect, concluding that sleep problems could cause approximately $15 \%$ of the $\mathrm{AD}$ cases in the population ${ }^{52}$.

Further research by Shi et al. ${ }^{43}$ also aimed to meta-analyse the predictive role of overall sleep disturbances in incident cognitive disorders. Moreover, they expanded the investigation, distinguishing different subtypes of sleep disturbances such as insomnia, sleep-disordered breathing and other sleep problems (e.g., excessive daytime sleepiness, sleep-related movement disorder, circadian rhythm sleep disorder, and nonspecific sleep problems). Furthermore, they analysed all-cause dementia, AD and vascular cognitive impairment separately. Eighteen out of a total of 12,926 papers were included in the meta-analysis. In the subgroup analysis, they found that insomnia increased the risk of $\mathrm{AD}$ but not vascular or all-cause dementia. On the contrary, sleep-disordered breathing was associated with both all-cause dementia, $\mathrm{AD}$ and vascular dementia ${ }^{43}$. These results, however, need to be confirmed by further studies specifically designed to better comprehend the difference of specific sleep disorders in the different types of dementia.

\section{Biomarkers}

In the last decade, biomarkers started to become part of the diagnostic criteria for $\mathrm{AD}^{53}$. Since then, some studies targeting sleep and $\mathrm{AD}$ began to include the biomarkers, improving the evidence of the link between sleep disturbances and $\mathrm{AD}$ pathology. A cross-sectional study of 95 healthy elderly, applying polysomnography and lumbar puncture, found an association between sleep-disordered breathing and cerebrospinal fluid (CSF) AD biomarkers ${ }^{54}$. Additionally, studies with other biomarkers, such as PET with amyloid and tau tracers, are being conducted.

\section{Potential mechanisms linking sleep disorders and AD risk}

Given the possible associations between sleep disturbances and $\mathrm{AD}$, studies aiming to uncover the underlying mechanisms have been conducted in animal models and humans. Sleep disorders may be related to the development of $\mathrm{AD}$ by reduction of non-REM sleep. Individuals in an awake state and sleep were studied with f-fluorodeoxyglucose (FDG-PET), which is a marker of metabolic activity in the brain. Their cerebral metabolic activity was similar in
REM sleep and wakefulness but declined substantially during slow-wave sleep (a decline of around 40\%) ) $^{2,6}$. Regionally, reductions of brain activity were located in subcortical and cortical (prefrontal, anterior cingulate and pre-cuneus cortices) regions ${ }^{6}$. This reduction is caused by the hyperpolarized silent state that happens to the neurons during slow-wave sleep. Synaptic activity is related to releases of amyloid- $\beta$ $(\mathrm{A} \beta)$ in the interstitial fluid ${ }^{2}$, which can be observed in vitro by the fluctuations of the $A \beta$ levels in murine ${ }^{55}$ models and humans ${ }^{56}$. The greater the duration of the awake state, the greater the $\mathrm{A} \beta$ concentration. Moreover, a further increase occurs in acute sleep deprivation experiments. Conversely, a decrease in the $A \beta$ levels occurs during sleep (especially in slow-wave sleep) ${ }^{56}$.

An important clearance mechanism of the brain's toxic substances was discovered recently and called the glymphatic system because of the central role of the glial cells on its physiology and the functional similarity to the lymphatic system. Rodent studies found that CSF is driven from the subarachnoid space to the periarterial spaces of the larger cerebral arteries, flowing along them as they branch into penetrating arteries. The CSF flows from the perivascular spaces to the brain interstitium facilitated by AQP4 transporters on the astrocyte's end-feet. The same transport mechanism occurs from the interstitium to the perivenous spaces and, in turn, CSF is dropped again in the subarachnoid space. Several mechanisms generate a convective bulk flow of interstitial fluid (ISF) from the periarterial to the perivenous space, through the interstitium, promoting washout of toxic substances accumulated in the brain, including $A \beta$ and tau protein ${ }^{57,58}$. A study published in 2012 revealed that a $60 \%$ expansion in the interstitium occurs during sleep, which facilitates convective exchange between CSF and ISF, promoting an increased $A \beta$ clearance during sleep ${ }^{7}$. Sleep disturbances, according to this mechanism, could lead to a decreased convective exchange between the CSF and ISF and, consequently, a decreased $\mathrm{A} \beta$ clearance.

Altogether, both potential consequences of disordered sleep states (an increased cerebral activity with increased $\mathrm{A} \beta$ accumulation and decreased functioning of the glymphatic exchanges with decreased $A \beta$ clearance) would lead to an increased concentration of soluble $A \beta$ in the ISF, which is associated with amyloid deposition ${ }^{59}$. The default mode network, which includes the pre-cuneus, lateral parietal and medial prefrontal brain regions, is more active during the rest state (when the individual is not attending to a specific task) and is the brain network with the highest levels of neuronal activity ${ }^{60}$. Not surprisingly, it is also the most susceptible brain region to accumulate $A \beta$ during the early progression of $\mathrm{AD}$.

Other possible risk factors in the development of neurodegeneration and $\mathrm{AD}$ are the circadian rhythm disorders. Despite the evidence indicating that the structures responsible for circadian rhythms are dysfunctional in patients with 


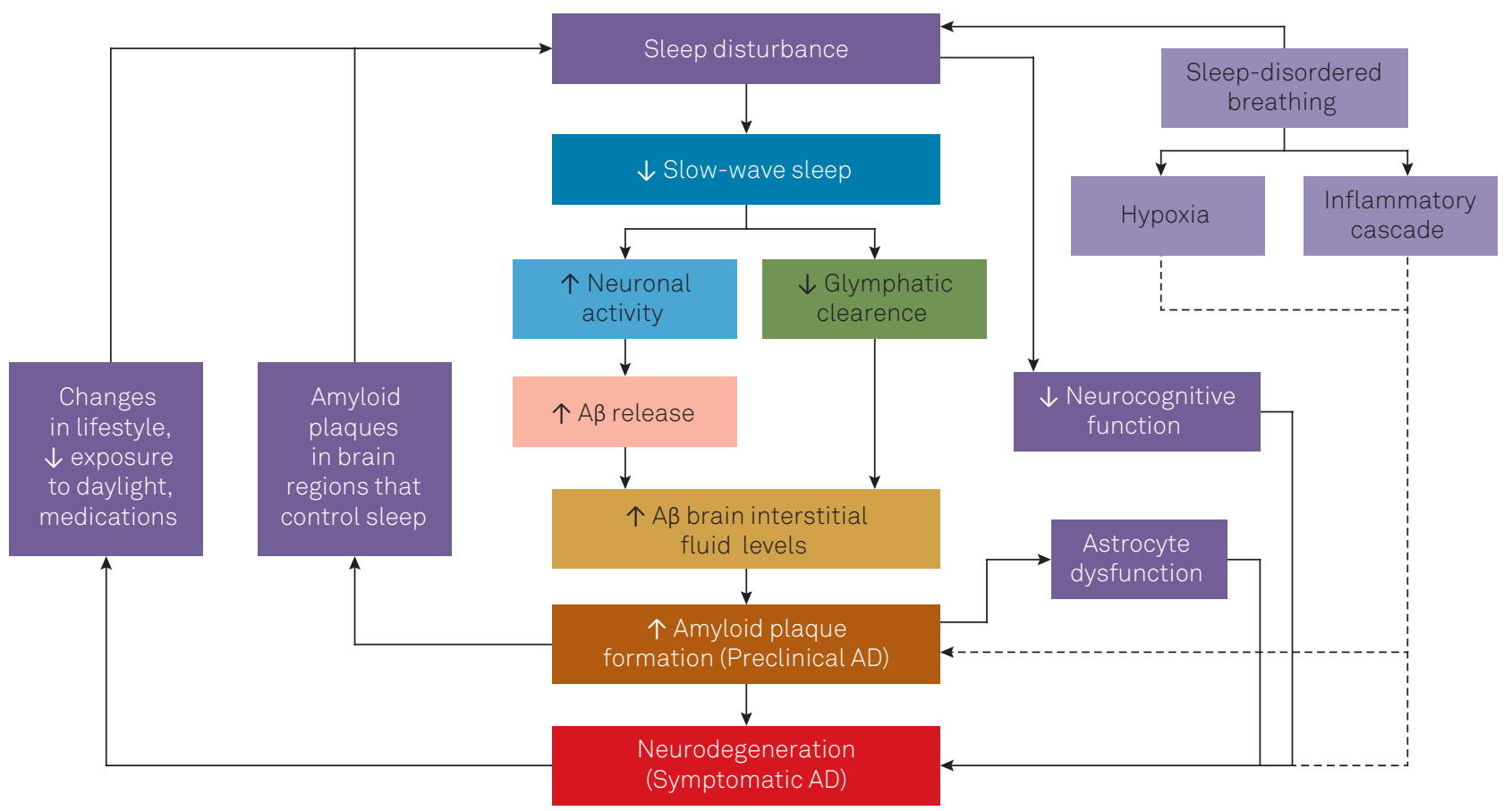

Figure 2. Graphic summary of the mechanisms of interaction between sleep disturbances and the pathogenesis of AD. Adapted from Ju et al. ${ }^{55}$. Sleep disturbances, by reduction of the slow-wave sleep, lead to an increased neuronal activity and reduced clearance of amyloid, resulting in increased $A \beta$ levels in the brain interstitial fluid, augmenting the risk of $A \beta$ plaque formation and astrocyte dysfunction, and, consequently, neurodegeneration and AD dementia.

definitive $\mathrm{AD}$ pathology, few studies have addressed the role of circadian rhythm misalignment in the pathogenesis of $\mathrm{AD}$. Some studies showed that the circadian clocks might regulate hippocampal-dependent learning9. Another, conducted in an animal model of "jetlag", showed that the animals exposed to circadian cycle disruption exhibited diminished hippocampal neurogenesis and impaired learning and memory, as well as increased blood inflammatory markers ${ }^{9}$. However, one of the best pieces of evidence underlying the circadian rhythm disruption in $\mathrm{AD}$ patients and the sundown syndrome is the degeneration of the suprachiasmatic nucleus ${ }^{61}$.

Finally, other negative consequences of disordered sleep have been studied, such as increases in neuronal oxidative stress, impairment in the blood-brain barrier and reduction in the hippocampal neurogenesis ${ }^{62}$. In Figure 2 we show a diagram with the proposed causal mechanisms.

Amyloid plaque formation occurs in various brain areas, including regions that control sleep, which would worsen the sleep disturbances, perpetuating the cycle. Likewise, many behavioural symptoms occur as $\mathrm{AD}$ dementia develops, such as changes in lifestyle, less exposure to daylight and use of polypharmacy, also interfering in the sleep quality of the demented individuals. On the other hand, the sleep disturbances impair their cognitive functions directly. Another cause of sleep disorders that could interfere directly in the pathogenesis of $\mathrm{AD}$ is sleep-disordered breathing, through hypoxia and inflammatory cascades.

\section{CONCLUSIONS}

The interface of neurodegenerative disorders and sleep is a topic with increasing relevance. Scientific evidence toward the refinement or stratification of dementia risk among subgroups of the older population, such as those with MCI, requires further investigation of potential predictors that are still under-recognized. Indicators of poor sleep or sleep disorders may play a role in the cascade of $\mathrm{AD}$ pathology and, consequently, increase the incidence of dementia among those with higher risk, including people with MCI. Therefore, the study of the sleep-related factors that occur precisely when $\mathrm{MCI}$ is established is of the utmost importance to the development of future interventions that could impact the natural history of $\mathrm{AD}$ and other neurodegenerative diseases.

\section{References}

1. Palma JA, Urrestarazu E, Iriarte J. Sleep loss as risk factor for neurologic disorders: a review. Sleep Med. 2013 Mar;14(3):229-36. https://doi.org/10.1016/j.sleep.2012.11.019
2. Ju YE, Lucey BP, Holtzman DM. Sleep and Alzheimer disease pathology: a bidirectional relationship. Nat Rev Neurol. 2014 Feb;10(2):115-9. https://doi.org/10.1038/nrneurol.2013.269 
3. Reynolds CF 3rd, Kupfer DJ, Taska LS, Hoch CC, Sewitch DE, Restifo K, et al. Sleep apnea in Alzheimer's dementia: correlation with mental deterioration. J Clin Psychiatry. 1985 Jul;46(7):257-61.

4. Schenck $\mathrm{CH}$, Bundlie SR, Ettinger MG, Mahowald MW. Chronic behavioral disorders of human REM sleep: a new category of parasomnia. Sleep. 1986 Jun;9(2):293-308. https://doi.org/10.1093/sleep/9.2.293

5. Uchiyama M, Isse K, Tanaka K, Yokota N, Hamamoto M, Aida $\mathrm{S}$, et al. Incidental Lewy body disease in a patient with REM sleep behavior disorder. Neurology. 1995 Apr;45(4):709-12. https://doi.org/10.1212/WNL.45.4.709

6. Dang-Vu TT, Schabus M, Desseilles M, Sterpenich V, Bonjean M, Maquet P. Functional neuroimaging insights into the physiology of human sleep [Internet]. Sleep. 2010 Dec;33(12):1589-603. https://doi.org/10.1093/sleep/33.12.1589

7. Xie L, Kang H, Xu Q, Chen MJ, Liao Y, Thiyagarajan M, et al. Sleep drives metabolite clearance from the adult brain. Science. 2013 Oct;342(6156):373-7. https://doi.org/10.1126/science.1241224

8. Mander BA, Winer JR, Jagust WJ, Walker MP. Sleep: a novel mechanistic pathway, biomarker, and treatment target in the pathology of Alzheimer's disease? Trends Neurosci. 2016 Aug;39(8):552-66. https://doi.org/10.1016/j.tins.2016.05.002

9. Musiek ES, Holtzman DM. Mechanisms linking circadian clocks, sleep, and neurodegeneration. Science. 2016 Nov;354(6315):1004-8. https://doi.org/10.1126/science.aah4968

10. Hita-Yanez E, Atienza M, Gil-Neciga E. L. Cantero J. Disturbed sleep patterns in elders with mild cognitive impairment: the role of memory decline and ApoE $\varepsilon 4$ genotype. Curr Alzheimer Res. 2012;9(3):290-7. https://doi.org/10.2174/156720512800107609

11. Hita-Yañez E, Atienza M, Cantero JL. Polysomnographic and subjective sleep markers of mild cognitive impairment [Internet]. Sleep (Basel). 2013 Sep;36(9):1327-34. https://doi.org/10.5665/sleep.2956

12. Hu M, Zhang P, Li C, Tan Y, Li G, Xu D, et al. Sleep disturbance in mild cognitive impairment: a systematic review of objective measures. Neurol Sci. 2017 Aug;38(8):1363-71. https://doi.org/10.1007/s10072-017-2975-9

13. Mander BA, Winer JR, Walker MP. Sleep and human aging. Neuron. 2017 Apr;94(1):19-36. https://doi.org/10.1016/j.neuron.2017.02.004

14. Ohayon MM, Carskadon MA, Guilleminault C, Vitiello MV. Meta-analysis of quantitative sleep parameters from childhood to old age in healthy individuals: developing normative sleep values across the human lifespan [Internet]. Sleep. 2004 Nov;27(7):1255-73. https://doi.org/10.1093/sleep/27.7.1255

15. Kang DW, Lee CU, Lim HK. Role of Sleep Disturbance in the trajectory of Alzheimer's disease. Clin Psychopharmacol Neurosci. 2017 May;15(2):89-99. https://doi.org/10.9758/cpn.2017.15.2.89

16. Moraes W, Piovezan R, Poyares D, Bittencourt LR, Santos-Silva R, Tufik S. Effects of aging on sleep structure throughout adulthood: a population-based study. Sleep Med. 2014 Apr;15(4):401-9. https://doi.org/10.1016/j.sleep.2013.11.791

17. Ancoli-Israel S, Ayalon L, Salzman C. Sleep in the elderly: normal variations and common sleep disorders. Harv Rev Psychiatry. 2008;16(5):279-86. https://doi.org/10.1080/10673220802432210

18. Münch M, Knoblauch V, Blatter K, Schröder C, Schnitzler C, Kräuchi $\mathrm{K}$, et al. Age-related attenuation of the evening circadian arousal signal in humans. Neurobiol Aging. 2005 Oct;26(9):1307-19. https://doi.org/10.1016/j.neurobiolaging.2005.03.004

19. Mander BA, Rao V, Lu B, Saletin JM, Lindquist JR, Ancoli-Israel S, et al. Prefrontal atrophy, disrupted NREM slow waves and impaired hippocampal-dependent memory in aging. Nat Neurosci. 2013 Mar;16(3):357-64. https://doi.org/10.1038/nn.3324
20. Clawson BC, Durkin J, Aton SJ. Form and Function of Sleep Spindles across the Lifespan. Neural Plast. 2016;2016(1):6936381. https://doi.org/10.1155/2016/6936381

21. Silva AA, Mello RG, Schaan CW, Fuchs FD, Redline S, Fuchs SC. Sleep duration and mortality in the elderly: a systematic review with meta-analysis. BMJ Open. 2016 Feb;6(2):e008119. https://doi.org/10.1136/bmjopen-2015-008119

22. Ohara T, Honda T, Hata J, Yoshida D, Mukai N, Hirakawa Y, et al. Association between daily sleep duration and risk of dementia and mortality in a Japanese community.J Am Geriatr Soc. 2018 Oct;66(10):1911-1918. https://doi.org/10.1111/jgs.15446

23. Harrison Y, Horne JA, Rothwell A. Prefrontal neuropsychological effects of sleep deprivation in young adults: a model for healthy aging? Sleep. 2000 Dec;23(8):1067-73. https://doi.org/10.1093/sleep/23.8.1f

24. Musiek ES, Bhimasani M, Zangrilli MA, Morris JC, Holtzman DM, Ju YS. Circadian rest-activity pattern changes in aging and preclinical Alzheimer disease. JAMA Neurol. 2018 May;75(5):582-90. https://doi.org/10.1001/jamaneurol.2017.4719

25. Petersen RC, Morris JC. Mild cognitive impairment as a clinical entity and treatment target. Arch Neurol. 2005 Jul;62(7):1160-3. https://doi.org/10.1001/archneur.62.7.1160

26. Beaulieu-Bonneau S, Hudon C. Sleep disturbances in older adults with mild cognitive impairment. Int Psychogeriatr. 2009 Aug;21(4):654-66. https://doi.org/10.1017/S1041610209009120

27. Bombois S, Derambure P, Pasquier F, Monaca C. Sleep disorders in aging and dementia.J Nutr Health Aging. 2010 Mar;14(3):212-7. https://doi.org/10.1007/s12603-010-0052-7

28. Bidzan M, Bidzan L, Bidzan-Bluma I. Neuropsychiatric symptoms and faster progression of cognitive impairments as predictors of risk of conversion of mild cognitive impairment to dementia. Arch Med Sci. 2017 Aug;13(5):1168-77. https://doi.org/10.5114/aoms.2017.68943

29. Muangpaisan W, Intalapaporn S, Assantachai P. Neuropsychiatric symptoms in the community-based patients with mild cognitive impairment and the influence of demographic factors. Int J Geriatr Psychiatry. 2008 Jul;23(7):699-703. https://doi.org/10.1002/gps.1963

30. Westerberg CE, Lundgren EM, Florczak SM, Mesulam MM, Weintraub S, Zee PC, et al. Sleep influences the severity of memory disruption in amnestic mild cognitive impairment: results from sleep self-assessment and continuous activity monitoring [Internet]. Alzheimer Dis Assoc Disord. 2010 Oct-Dec;24(4):325-33. https://doi.org/10.1097/WAD.0b013e3181e30846

31. Naismith SL, Rogers NL, Hickie IB, Mackenzie J, Norrie LM, Lewis SJ. Sleep well, think well: sleep-wake disturbance in mild cognitive impairment. J Geriatr Psychiatry Neurol. 2010 Jun;23(2):123-30. https://doi.org/10.1177/0891988710363710

32. Peter-Derex L, Yammine P, Bastuji H, Croisile B. Sleep and Alzheimer's disease. Sleep Med Rev. 2015 Feb;19:29-38. https://doi.org/10.1016/j.smrv.2014.03.007

33. Vitiello M V, Prinz PN, Williams DE, Frommlet MS, Ries RK. Sleep disturbances in patients with mild-stage Alzheimer's disease. J Gerontol. 1990;45(4):M131-8.

34. Petit D, Gagnon JF, Fantini ML, Ferini-Strambi L, Montplaisir J. Sleep and quantitative EEG in neurodegenerative disorders. J Psychosom Res. 2004 May;56(5):487-96. https://doi.org/10.1016/j.jpsychores.2004.02.001

35. Bliwise DL. Sleep in normal aging and dementia [Internet]. Sleep. 1993 Jan;16(1):40-81. https://doi.org/10.1093/sleep/16.1.40

36. Bliwise DL. Sleep disorders in Alzheimer's disease and other dementias. Clin Cornerstone. 2004;6(1 Suppl 1A):S16-28. https://doi.org/10.1016/S1098-3597(04)90014-2 
37. Walsh CM, Blackwell T, Tranah GJ, Stone KL, Ancoli-Israel S, Redline $S$, et al. Weaker circadian activity rhythms are associated with poorer executive function in older women. Sleep (Basel). 2014 Dec;37(12):2009-16. https://doi.org/10.5665/sleep.4260

38. Foley D, Monjan A, Masaki K, Ross W, Havlik R, White L, et al. Daytime sleepiness is associated with 3-year incident dementia and cognitive decline in older Japanese-American men. J Am Geriatr Soc. 2001 Dec;49(12):1628-32. https://doi.org/10.1111/j.1532-5415.2001.49271.x

39. Elwood PC, Bayer AJ, Fish M, Pickering J, Mitchell C, Gallacher JE. Sleep disturbance and daytime sleepiness predict vascular dementia.J Epidemiol Community Health. 2011 Sep;65(9):820-4. https://doi.org/10.1136/jech.2009.100503

40. Virta JJ, Heikkilä K, Perola M, Koskenvuo M, Räihä I, Rinne JO, et al. Midlife sleep characteristics associated with late life cognitive function. Sleep (Basel). 2013 Oct;36(10):1533-41. https://doi.org/10.5665/sleep.3052

41. Chen JC, Espeland MA, Brunner RL, Lovato LC, Wallace RB, Leng $X$, et al. Sleep duration, cognitive decline, and dementia risk in older women. Alzheimers Dement. 2016 Jan;12(1):21-33. https://doi.org/10.1016/j.jalz.2015.03.004

42. Potvin O, Lorrain D, Forget H, Dubé M, Grenier S, Préville M, et al. Sleep quality and 1-year incident cognitive impairment in community-dwelling older adults [Internet]. Sleep (Basel). 2012 Apr;35(4):491-9. https://doi.org/10.5665/sleep.1732

43. Shi L, Chen SJ, Ma MY, Bao YP, Han Y, Wang YM, et al. Sleep disturbances increase the risk of dementia: a systematic review and meta-analysis. Sleep Med Rev. 2018 Aug;40:4-16. https://doi.org/10.1016/j.smrv.2017.06.010.

44. Cohen-Zion M, Stepnowsky C, Marler, Shochat T, Kripke DF, Ancoli-Israel S. Changes in cognitive function associated with sleep disordered breathing in older people [Internet]. J Am Geriatr Soc. 2001 Dec;49(12):1622-7. https://doi.org/10.1111/j.1532-5415.2001.49270.x

45. Benito-León J, Bermejo-Pareja F, Vega S, Louis ED. Total daily sleep duration and the risk of dementia: a prospective population-based study. Eur J Neurol. 2009 Sep;16(9):990-7. https://doi.org/10.1111/j.1468-1331.2009.02618.x

46. Loerbroks A, Debling D, Amelang M, Stürmer T. Nocturnal sleep duration and cognitive impairment in a population-based study of older adults. Int J Geriatr Psychiatry. 2010 Jan;25(1):100-9.

47. Tranah GJ, Blackwell T, Stone KL, Ancoli-Israel S, Paudel ML, Ensrud KE, et al.; SOF Research Group. Circadian activity rhythms and risk of incident dementia and mild cognitive impairment in older women. Ann Neurol. 2011 Nov;70(5):722-32. https://doi.org/10.1002/ana.22468

48. Blackwell T, Yaffe K, Laffan A, Ancoli-Israel S, Redline S, Ensrud KE, et al. Associations of objectively and subjectively measured sleep quality with subsequent cognitive decline in older communitydwelling men: the MrOS sleep study. Sleep (Basel). 2014 Apr;37(4):655-63. https://doi.org/10.5665/sleep.3562

49. Yaffe K, Laffan AM, Harrison SL, Redline S, Spira AP, Ensrud $K E$, et al. Sleep-disordered breathing, hypoxia, and risk of mild cognitive impairment and dementia in older women. JAMA. 2011 Aug;306(6):613-9. https://doi.org/10.1001/jama.2011.1115
50. Pase MP, Himali JJ, Grima NA, Beiser AS, Satizabal CL, Aparicio $\mathrm{HJ}$, et al. Sleep architecture and the risk of incident dementia in the community. Neurology. 2017 Sep;89(12):1244-50. https://doi.org/10.1212/WNL.0000000000004373

51. de Almondes KM, Costa MV, Malloy-Diniz LF, Diniz BS. Insomnia and risk of dementia in older adults: systematic review and meta-analysis. J Psychiatr Res. 2016 Jun;77:109-15. https://doi.org/10.1016/j.jpsychires.2016.02.021

52. Bubu OM, Brannick M, Mortimer J, Umasabor-Bubu O, Sebastião YV, Wen Y, et al. Sleep, cognitive impairment, and Alzheimer's disease: a systematic review and meta-analysis. Sleep (Basel). 2017 Jan;40(1): https://doi.org/10.1093/sleep/zsw032

53. Jack CR Jr, Albert M, Knopman DS, Mckhann GM, Sperling RA, Carillo M, et al. Introduction to revised criteria for the diagnosis of Alzheimer's disease: National Institute on Aging and the Alzheimer Association Workgroups. Alzheimer's Dement J Alzheimer's Assoc. 2011 May;7(3):257-62. https://doi.org/10.1016/j.jalz.2011.03.004

54. Osorio RS, Ayappa I, Mantua J, Gumb T, Varga A, Mooney AM, et al. Interaction between sleep-disordered breathing and apolipoprotein E genotype on cerebrospinal fluid biomarkers for Alzheimer's disease in cognitively normal elderly individuals [Internet]. Neurobiol Aging. 2014 Jun;35(6):1318-24. https://doi.org/10.1016/j.neurobiolaging.2013.12.030

55. Kang J-E, Lim MM, Bateman RJ, Lee JJ, Smyth LP, Cirrito JR, et al. Amyloid-dynamics are regulated by orexin and the sleep-wake cycle. Science. 2009 Sep;326(5955):1005-7. https://doi.org/10.1126/science.1180962

56. Huang Y. Effect of age and amyloid deposition on a $\beta$ dynamics in the human central nervous system. Arch Neurol. 2012;69(1):51-8. https://doi.org/10.1001/archneurol.2011.235

57. Rasmussen MK, Mestre H, Nedergaard M. The glymphatic pathway in neurological disorders. Lancet Neurol. 2018 Nov;17(11):1016-24. https://doi.org/10.1016/S1474-4422(18)30318-1

58. Jessen NA, Munk AS, Lundgaard I, Nedergaard M. The glymphatic system: a beginner's guide. Neurochem Res. 2015 Dec;40(12):2583-99. https://doi.org/10.1007/s11064-015-1581-6

59. Bero AW, Yan P, Roh JH, Cirrito JR, Stewart FR, Raichle ME, et al. Neuronal activity regulates the regional vulnerability to amyloid- $\beta$ deposition. Nat Neurosci. 2011 Jun;14(6):750-6. https://doi.org/10.1038/nn.2801

60. Ju YE, McLeland JS, Toedebusch CD, Xiong C, Fagan AM, Duntley SP, et al. Sleep quality and preclinical Alzheimer disease. JAMA Neurol. 2013 May;70(5):587-93. https://doi.org/10.1001/jamaneurol.2013.2334

61. Zhou L, Gao Q, Nie M, Gu JL, Hao W, Wang L, et al. Degeneration and energy shortage in the suprachiasmatic nucleus underlies the circadian rhythm disturbance in ApoE-/- mice: implications for Alzheimer's disease. Sci Rep. 2016 Nov;6(April):36335. https://doi.org/10.1038/srep36335

62. Kent BA, Mistlberger RE. Sleep and hippocampal neurogenesis: implications for Alzheimer's disease. Front Neuroendocrinol. 2017 Apr;45:35-52. https://doi.org/10.1016/j.yfrne.2017.02.004 